\title{
Impact of the “Anisakis C.Re.N.A.” APP
}

\author{
Vincenzo Ferrantelli, Antonella Costa, Stefania Graci, Gaetano Cammilleri, Maria Drussilla Buscemi, Rosaria \\ Collura, Gianluigi Maria Lo Dico, Valentina Cumbo, Francesca Ornella Assiria, Michele Chetta, Andrea \\ Macaluso \& Antonio Vella
}

Centro di Referenza Nazionale per le Anisakiasi, Istituto Zooprofilattico Sperimentale della Sicilia “A. Mirri”, Via G. Marinuzzi 3, 90129 Palermo, Italy

\begin{abstract}
S
Anisakiasis is a zoonotical disease provoked by the nematodes belonged to the Anisakidae family. The trend of Anisakiasis cases increases continously, therefore a constant awareness of this topic is essential for the consumers' protection. An application for smartphones was carried out by the National Centre for Anisakiasis for this purpose. This work aimed at evaluate the impact of "Anisakis CReNA" app for consumers by the implementation of a survey. The results obtained revealed that the app developed has reached the goal established giving easy to understand and easy to consult information for the protection of consumers regarding the risks related to these parasites.
\end{abstract}

KEY WORDS

Anisakis; National Reference Centre; health.

Received 22.11.2019; accepted 10.12.2019; published online 20.12.2019

Proceedings of the 4th International Congress on Biodiversity "Man, Natural Habitats and Euro-Mediterranean Biodiversity", November 17th-19th, 2017 - Malta

The application "Anisakis C.Re.N.A.” was developed by the National Reference Centre for Anisakiasis (C.Re.N.A.) of Istituto Zooprofilattico Sperimentale della Sicilia, with the aim to divulging information about the health risk related to Anisakis parasites in fish products. There are several recommendations on the consumption and correct preparation of fish-based recipes and detailed information on their related diseases such as Anisakiasis with related photos and videos. It is possible to download it freely for the Android and iOS platforms.

Today, there are a lot of appllications which give a valuable contribution to ensure food safety giving the widespread use of smartphones or tablet. The app is proved to be very smart and easy because of its subdivision in topic icons. The homepage is divided as follow: informations about CReNA, Anisakis, Anisakiasis, normative and cooking recipes.

The digital devices that are use daily are the best tools for communication purpose concerning lifestyle areas, comprising cookery. For these reasons, the app contain delicious fish-based recipes with all the recommendations for consumers to ensure the maintenance of food safety. The work aimed to evaluate the impact of "Anisakis CReNA" app for consumers by the implementation of a survey.

A total of three hundred persons were contacted in supermarkets, fish street vendors and hotel management Schools of Sicily and primed to download and evaluate the app. Of these, 150 downloaded the app and evaluate it with a good feedback. A Monte Carlo test was conducted in order to have a clear estimation of the satisfaction grade subdivided by person category.

The test was carried out by R 2.1.4 software. The users that downloaded the Anisakis app were doctors, housewives and food sector operators. $80 \%$ of housewives showed a valuable interest on the Anisakis 
topic, asking more information about the good practices of cooking fish products; the remaining $20 \%$ alerted themselves declaring to avoid the consumption of food products. Of these, over $90 \%$ were females between 50 and 60 years old. All the doctors gave a $100 \%$ positive feedback of the app highlighting the need to emphasize the information of the app to the consumers. $90 \%$ of the food sectors operators showed high interest to the information included in the app; the remaining $10 \%$ declared that the information of the app could be dangerous for the fish products sectors because are too alarmist and able to deviate the consumers. $94 \%$ of the users declared the app very useful, smart and easy. Finally, the results obtained revealed that the app developed has reached the goal established giving easy to understand and easy to consult information for the protection of consumers regarding the risks related to these parasites.

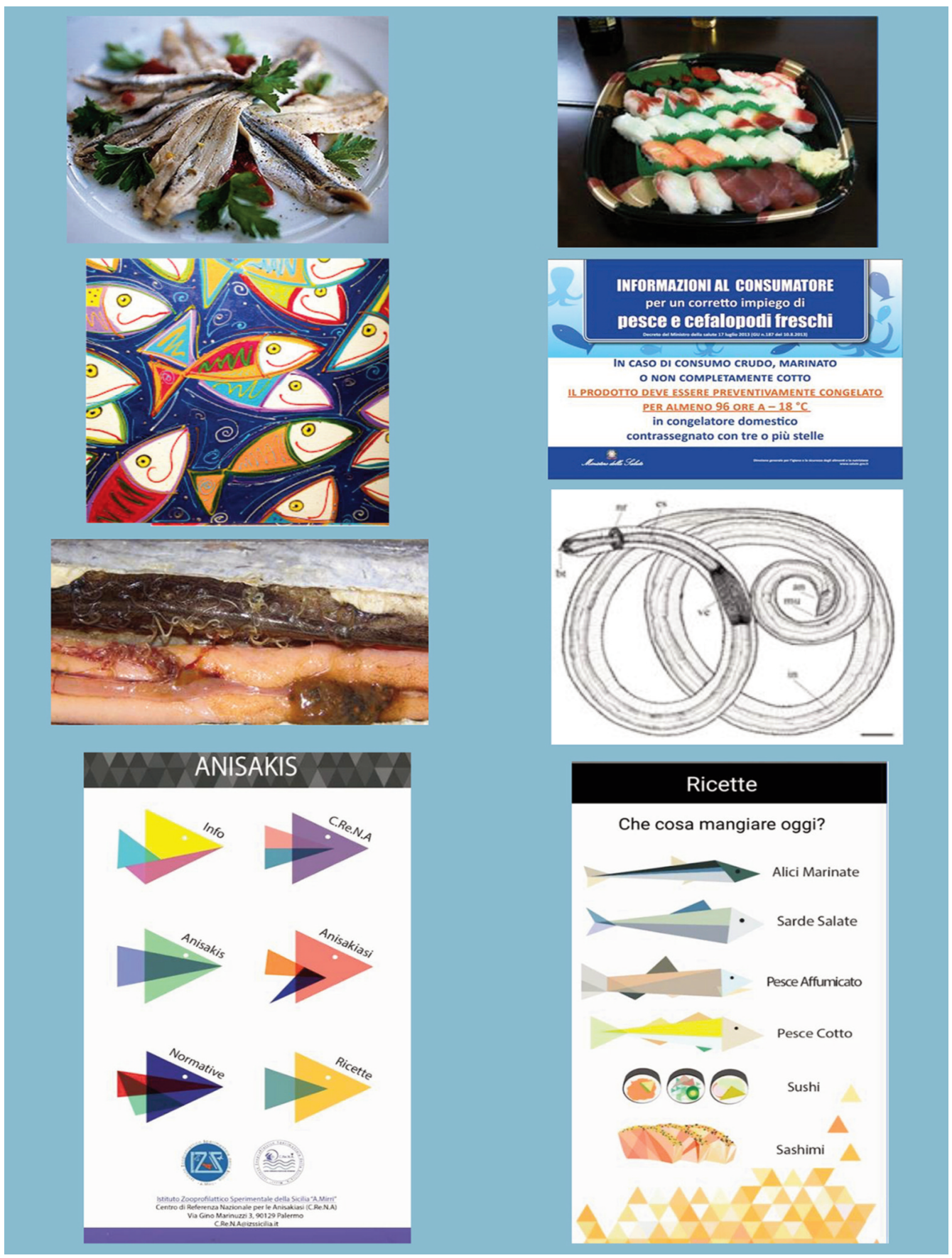

